\title{
SNMMI Comment on ASCO 2013 "Choosing Wisely" Recommendation on Use of PET/CT in Recurrent Cancer Surveillance
}

$\mathbf{T}$ he Society of Nuclear Medicine and Molecular Imaging (SNMMI) would like to comment on the recent publication of the American Society of Clinical Oncology (ASCO) Top Five List in Oncology as part of the Choosing Wisely Campaign. Specifically, we wish to comment on recommendation 3, which states: "Avoid using PET or PET-CT scanning as part of routine followup care to monitor for cancer recurrence in asymptomatic patients who have finished initial treatment to eliminate the cancer unless there is high-level evidence that such imaging will change the outcome." The recommendation further states that monitoring of recurrence with PET/CT does not improve outcomes and that falsepositive tests can lead to unnecessary and invasive procedures (1).

The SNMMI feels that these statements are overly broad and are subject to misinterpretation by the oncologic community. First and foremost, it must be clarified that the subject of this recommendation by ASCO is PET/CT using ${ }^{18} \mathrm{~F}-\mathrm{FDG}$. There are other radiotracers for oncologic imaging with PET/CT, including Food and Drug Administration-approved radiotracers such as ${ }^{18} \mathrm{~F}$-fluoride for bone imaging and ${ }^{11} \mathrm{C}$-choline for imaging of prostate cancer. With the emergence of new radiotracers, we encourage the medical community to communicate with a greater degree of accuracy, specifying the type of PET/CT scan under discussion (e.g., ${ }^{18}$ F-FDG PET/CT).

With regard to false-positive results, this is an issue with any diagnostic procedure. The authors of the recommendation supply no references to support the implication that ${ }^{18} \mathrm{~F}-\mathrm{FDG}$ PET/CT is particularly more prone to false-positive results than are other imaging tests such as CT or MR imaging. In the setting of disease detection and surveillance, it must also be recognized that the performance characteristics of a given diagnostic procedure must match the clinical use and that false-positive rates (i.e., high sensitivity) are sometimes desirable. In screening mammography, for example, it would be beneficial to have high sensitivity over high specificity and to rely on the postscreening workup to differentiate true- from false-positive cases. Other factors obviously come into the discussion, including cost, comparative effectiveness, and accessibility, all missing from the ASCO recommendation.

Another issue is the statement that "Until high-level evidence demonstrates that routine surveillance with PET or PET-CT scans helps prolong life or promote well-being after treatment for a specific type of cancer, this practice should not be performed." There are existing definitions of levels of evidence-for example, those put forward by the National Comprehensive Cancer Network-but

Received Feb. 28, 2014; revision accepted Mar. 5, 2014.

For correspondence or reprints contact: Hossein Jadvar, University of Southern California, 2250 Alcazar St., CSC 102, Los Angeles, CA 90033.

E-mail: jadvar@med.usc.edu

Published online Mar. 24, 2014.

COPYRIGHT (C) 2014 by the Society of Nuclear Medicine and Molecular Imaging, Inc.

DOI: $10.2967 /$ jnumed.114.139683 the authors of the recommendation supply neither a definition nor a reference. In the absence of such a definition, the term high-level evidence becomes a moving target, and difficult to achieve.

In fact, there are studies in the literature promoting the use of "surveillance" ${ }^{18}$ F-FDG PET/CT for selected tumor types, showing good test performance characteristics and improvement of patient outcomes. For example, in patients with cancers of the head and neck, disease detection with ${ }^{18}$ F-FDG PET/CT leads to improved outcomes $(2,3)$. Similarly, in patients with colorectal carcinoma with rising carcinoembryonic antigen levels and negative findings on conventional imaging, ${ }^{18} \mathrm{~F}-\mathrm{FDG}$ PET/CT shows incremental benefit in the detection of the site of recurrence, which could lead to directed therapy such as stereotactic radiotherapy (4).

It is also established in the literature that there are scenarios in which surveillance imaging with ${ }^{18} \mathrm{~F}-\mathrm{FDG}$ PET/CT provides no incremental benefit to the patient, such as in patients with certain types of lymphoma $(5,6)$. To the point of emphasis on outcomes, we highlight an article in which surveillance imaging with ${ }^{18} \mathrm{~F}-\mathrm{FDG}$ PET/CT in patients with advanced melanoma was shown to detect disease earlier than any other imaging procedure, yet despite the early detection there was no impact on overall patient survival (7).

The recommendation also refers to policy documents from the Centers for Medicare and Medicaid Services and Cancer Care Ontario. These governmental bodies are directly or indirectly concerned with the economic impact of coverage of ${ }^{18} \mathrm{~F}-\mathrm{FDG}$ PET/CT scans and as a result could be perceived as having a disincentive to promote their use. The ASCO recommendation also refers to a practice guideline issued by the European Society of Medical Oncology that discusses all aspects of the care of the patient with colon cancer. In fact, close review of this article reveals that ${ }^{18} \mathrm{~F}-\mathrm{FDG}$ $\mathrm{PET} / \mathrm{CT}$ is not mentioned specifically in the section on surveillance. The authors merely state, "other laboratory and radiological examinations are of unproven benefit...."

The SNMMI invites ASCO to collaborate on a framework for the incorporation of imaging into clinical trials, such that all involved parties could consider the results "high-level evidence." In such a way, the societies could lead the way in defining and promoting the use of high-quality and impactful imaging and appropriately and specifically discourage the use of imaging when not justified by data. Such collaboration will better serve our patients and the physicians who care for them, by providing data-driven recommendations for the use of imaging in the cancer patient.

\section{REFERENCES}

1. Schnipper LE, Lyman GH, Blayney DW, et al. American Society of Clinical Oncology 2013 top five list in oncology. J Clin Oncol. 2013;31:4362-4370.

2. Kostakoglu L, Fardanesh R, Posner M, et al. Early detection of recurrent disease by FDG-PET/CT leads to management changes in patients with squamous cell cancer of the head and neck. Oncologist. 2013;18:1108-1117. 
3. Dunsky KA, Wehrmann DJ, Osman MM, et al. PET-CT and the detection of the asymptomatic recurrence or second primary lesions in the treated head and neck cancer patient. Laryngoscope. 2013;123:2161-2164.

4. Makis W, Kurzencwyg D, Hickeson M. ${ }^{18}$ F-FDG PET/CT superior to serum CEA in detection of colorectal cancer and its recurrence. Clin Imaging. 2013;37:1094-1097.

5. Eissa HM, Allen CE, Kamdar K, et al. Pediatric Burkitt's lymphoma and diffuse B-cell lymphoma: are surveillance scans required? Pediatr Hematol Oncol. October 2, 2013 [Epub ahead of print].

6. Cheah CY, Hofman MS, Dickinson M, et al. Limited role for surveillance PET-CT scanning in patients with diffuse large B-cell lymphoma in complete metabolic remission following primary therapy. Br J Cancer. 2013;109:312-317.

7. Rueth NM, Xing Y, Chiang YJ, et al. Is surveillance imaging effective for detecting surgically treatable recurrences in patients with melanoma? A comparative analysis of stage-specific surveillance strategies. Ann Surg. October 3, 2013 [Epub ahead of print].
Eric M. Rohren

Immediate Past President, SNMMI PET Center of Excellence M.D. Anderson Cancer Center, Houston, Texas

Gary L. Dillehay

President, SNMMI

Northwestern University, Chicago, Illinois

Hossein Jadvar

President, SNMMI PET Center of Excellence

Vice President-Elect, SNMMI

University of Southern California, Los Angeles, California 